\title{
Proceedings of the meeting of the Association of British Neurologists, Manchester, 18-20 April 1985
}

FAMILY STUDIES IN MYASTHENIA GRAVIS RA Metcalf, L Kerzin-Storrar, PA Dyer, $P$ Wilson, G Kowalska. Manchester Royal Infirmary.

Myasthenia gravis is probably aetiologically heterogeneous and a range of factors including sex, age at onset, thymus histology and HLA type may help to sub-divide the disease. We have studied a series of 44 patients and their first and second degree relatives (in all 192 individuals) in an effort to identify those factors which can best define such subgroups. In addition to analysing clinical parameters, family studies enabled us to establish HLA-A,B and DR bearing haplotypes, family history of autoimmune disease and the presence of autoantibodies in relatives. In all patients we confirmed the previous reported increases in HLA-B8 and DR-3. This increase is most marked in young onset, female patients without thymoma. We also confirmed an increase in HLA-A 3 in older onset patients. In addition we report an increased frequency of HLAB5 which is significant in the young onset patients (26\% v. $5 \%$ of controls; pc 0.026$)$. Previous reports of non-family data have suggested that the well recognised haplotype HLA-A1, B8, DR3 could be increased in myasthenia gravis. However, our established haplotype data cannot confirm these speculations as the increases in frequency of B8 and DR3 are independent of the A1, B8, DR3 haplotype. An additional autoimmune disease was present in four patients, all of whom were female, and HLA-DR3 positive. A positive family history of auto-immune disease was confirmed in $13(30 \%)$ patients. All affected relatives were related to the patients through the maternal line. The structure of the pedigrees is consistent with an $\mathrm{X}$-linked factor contributing to autoimmune disease liability.

\section{IMMUNOGLOBULIN SERUM FACTOR IN} ACETYLCHOLINE RECEPTOR ANTIBODY NEGATIVE MYASTHENIA GRAVIS IMPAIRS NEUROMUSCULAR TRANSMISSION

S Mossman (introduced), A Vincent (introduced) and J Newsom-Davis. Department of Neurological Science, Royal Free Hospital School of Medicine and Institute of Neurology, London

$10 \%$ of patients with typical symptoms of myasthenia gravis (MG) have no detectable antibody to acetylcholine receptor (AChR) yet may improve following plasma exchange. We compared the effects in $18-20 \mathrm{~g}$ BKTO mice of intraperitoneal injection $(60$ $\mathrm{mg}$ /day for 3 days) of crude immunoglobulin (Ig) plasma fractions obtained from 12 antiAChR negative MG patients, and of control human Ig. Neuromuscular transmission was assessed in vitro by measuring the decrement in isometric twitch tension evoked in the diaphragm by supramaximal phrenic nerve stimulation at $3 \mathrm{~Hz}$, in the presence of 0.8 $\mu \mathrm{g} / \mathrm{ml}$ of d-tubocurarine. Receptor loss was measured by ${ }^{125} \mathrm{I}-\mathrm{a}-\mathrm{BuTx}$ binding, and $\mathrm{Ig}$ antibody-bound by immunoprecipitation of solubilised AChR.

Anti-AChR negative Ig caused a significant decrement compared with control human $\operatorname{Ig}(\mathrm{P}<0.001)$. Only 2 of 10 preparations inducing a substantial decrement had evidence of anti-AChR bound to receptors in situ (which had not been detected using solubilised $\mathrm{AChR}$ ), and one of these and one other showed receptor loss of up to $35 \%$. Thus the 7 other preparations impaired neuromuscular transmission in mice without evidence of AChR loss or antibody bound. We conclude that anti-AChR antibody negative MG sera contain Ig factor(s) that interfere with neuromuscular transmission by a mechanism that may differ from that of antiAChR.

\section{SLOWED MOTOR CONDUCTION IN THE LUMBO-SACRAL MOTOR ROOTS IN CAUDA EQUINA LESIONS}

Michael Swash and Steven J Snooks. St Marks Hospital and The London Hospital, London

Diagnosis of cauda equina lesions by myelography is justifiable only if surgical treatment is likely. Adequate methods for electrophysiological diagnosis vould improve management. We have measured motor latencies to the external anal sphincter muscles from spinal stimulation at $\mathrm{Ll}$ and L4 vertebral levels ( $500-1500 \mathrm{~V} ; 0.5 \mathrm{~ms}$; T.C. $50 \mu \mathrm{s})$, and from intra-anal stimulation of the pudendal nerves in 10 patients with cauda equina lesions, including ependymoma, spinal stenosis and arachnoiditis. In four patients with low back pain these studies were normal. In 32 control subjects, matched for age and sex, mean L1 and L4 motor latencies were $5.5 \pm 0.4 \mathrm{~ms}$ and $4.4 \Phi$ ms. In the 10 patients with cauda equinæd disease these latencies were $7.2 \pm 0.8 \mathrm{~ms}$ and $4.6 \pm 0.9 \mathrm{~ms}$, a significant increase in the $\mathrm{L}$ latency. The L1/L4 Latency ratios (spin latency ratios) were $1.72 \pm 0.13$ in caud equina patients and $1.3 \pm 0.1$ in norma subjects. Pudendal terminal motor latencies were normal. Single fibre EMG fibre densi ties (normal $1.5 \pm 0.19$ ) in the external and sphincter were increased in patients wi雨 cauda equina lesions $(1.73 \pm 0.28)$. In women with idiopathic neurogenic anorect incontinence there was evidence of rein nervation in the external anal sphincten (fibre density $1.98 \pm 0.31$ ) and puborectalis muscles (fibre density $1.74 \pm 0.4$ ). The pudendal nerve terminal motor latencies we increased in all of these patients and motor conduction from $\mathrm{Ll}$ and $\mathrm{L} 4$ to the pelve floor muscle were normal in all but seven of them. Slowing of motor conduction in the cauda equina is thus a sensitive test of defnage to these intraspinal motor roots than contributes to management.

$\mathrm{CO}_{2}$ RESPONSIVENESS FOLLOWING CEREBROVASCULAR RECONSTRUCTIVE SURGERY

I Bone, AD Mendelow, J Patterson, I Millan, R Quinn. Institute of Neurologicis Sciences, Glasgow

In patients with occlusive disease of the cap otid system the response of the cerebral cis culation to inhaled carbon dioxide gives a indication of the collateral reserve capacity The purpose of the present study was to do termine whether or not $\mathrm{CO} 2$ responsivenes improved following endarterectomy extracranial/intracranial (EC/IC) arteriä bypass surgery. Cerebral blood flow wag measured by the ${ }^{133}$ Xenon inhalation method. $\mathrm{CBF}$ and $\mathrm{CO} 2$ responsiveness werè measured before surgery and at least si weeks following surgery. Endarterectomy was performed in 29 patients and EC/IC by pass in 18 patients.

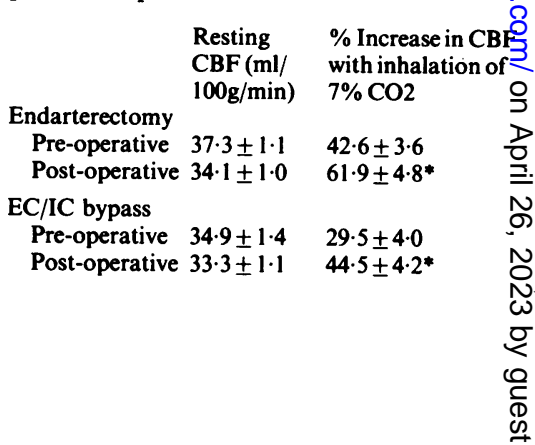


There was a significant increase $\left({ }^{*}\right)$ in the post-operative $\% \mathrm{CO} 2$ response in both endarterectomy and EC/IC bypass patients. The lower $\%$ increase in the pre-operative $\mathrm{CO} 2$ response in the patients with $\mathrm{EC} / \mathrm{IC}$ bypass may be accounted for by the fact that these patients had an internal carotid occlusion, while those with endarterectomy had a stenosis. These studies indicate that $\mathrm{CO} 2$ responsiveness returns towards normal after both endarterectomy and EC/IC bypass.

NEUROLOGICAL COMPLICATIONS OF CORONARY ARTERY BYPASS SURGERY

Pamela J Shaw, D Bates, NEF Cartlidge, DA Shaw. Department of Neurology, University of Newcastle upon Tyne

A prospective study of the neurological and neuropsychological complications occurring in patients undergoing elective coronary artery bypass surgery, has been undertaken. Three hundred and twelve patients have been evaluated over a 12 month period from September 1983 to August 1984. The patients underwent detailed neurological assessment in the two days prior to surgery and daily in the early post-operative period. They have been reviewed on an outpatient basis at intervals during the first six months after surgery.

Early post-operative neurological complications developed in $191 / 312$ patients $(61 \%)$ and include the following:-

1. Death due to extensive cerebral hypoxic damage occurred in one patient $(0 \cdot 3 \%)$.

2. Prolonged depression of conscious level was observed in 10 patients $(3 \cdot 2 \%)$.

3. Major or minor stroke occurred in 24 patients $(7 \cdot 7 \%)$.

4. Ophthalmological abnormalities including retinal infarction, retinal embolisation or other abnormality of vision occurred in 78 patients $(25 \%)$.

5 . Primitive reflexes developed in 123 patients $(39 \cdot 4 \%)$.

6. Post operative psychosis was observed in four patients $(1 \cdot 3 \%)$.

7. Peripheral nerve damage (most commonly brachial plexopathy) occurred in 37 patients $(11 \cdot 8 \%)$.

Although such a high proportion of the cohort developed detectable post-operative neurological abnormalities, in many cases these were not functionally significant. $138 / 191$ patients had no important functional disability while in hospital. 48/191 had some difficulty in managing their everyday activities and were considered to be mildly disabled. Severe disability or death from neurological causes occurred in only 5 patients.
HOW OFTEN IS CEREBRAL INFARCTION NOT DUE TO ARTHEROMA?: THE OXFORDSHIRE COMMUNITY STROKE PROJECT

PAG Sandercock, CP Warlow, LL Jones. University Department of Clinical Neurology, Oxford

The Oxfordshire Community Stroke Project is a prospective register of all new cases of stroke and transient ischaemic attack (TIA) in a defined population. In its first two years we registered 244 cases of first cerebral infarction (CI) verified by computed tomographic scanning (CT) or necropsy.

Of the 244 patients $42(17 \%)$ were apparently free, at the time of the stroke, of risk factors for CI (Previously diagnosed hypertension, TIA, atrial fibrillation, ischaemic heart disease, peripheral vascular disease and diabetes mellitus) and had no cardiac source of embolism. However, in 33 of the 42 , other factors became apparent; hypertension first diagnosed on follow-up after the stroke in 15 non-atheromatous conditions which might have caused the $\mathrm{CI}$ in 8 extreme age (over 80 years) in 10 .

The non-atheromatous conditions present in the 8 patients free of risk factors for $\mathrm{CI}$ were: arteritis in one, migrainous' $\mathrm{CI}$ in three and recent major surgery in one. There was one case each of:- thrombotic state associated with acute inflammatory bowel disease, arterial trauma and presumed invasion of the carotid artery by carcinoma.

In this community-based series, 217 $(88.9 \%)$ cases of $\mathrm{CI}$ had risk factors for $\mathrm{CI}$ or evidence of vascular disease in the head or legs, 18 others $(3.3 \%)$ had only nonatheromatous conditions and ten $(4 \cdot 1 \%)$ were aged over 80 . In only nine cases (3.7\%), therefore, was no cause for CI apparent.

THE ACCURACY OF THE GUY'S HOSPITAL SCORE FOR THE CLINICAL DIAGNOSIS OF ACUTE STROKE

CMC Allen, ${ }^{*}$ PAG Sandercock, $\dagger$ CP Warlow. $\dagger$ Departments of Neurology of Charing Cross Hospital* and Oxford University $\dagger$

The Guy's Hospital Stroke Diagnostic Score (GHSDS) was tested using the clinical data from 228 patients with acute stroke in the Oxfordshire Community Stroke Project (OCSP). The diagnosis was verified in each case by CT scan or necropsy. The optimum cut-off point on the score was determined for the differentiation of cerebral infarction from haemorrhage. This was found to be the same for both the OCSP patients and the original Guy's Hospital sample. Using this cut-off on the score $34 / 42(81 \%)$ of the OCSP patients with cerebral haemorrhage were correctly diagnosed and 145/186 of those with infarctions (overall predictive value $78 \%$ ). Four patients with cerebral haemorrhage had atrial fibrillation, of whom two were misdiagnosed by the score. Three of the 25 patients with atrial fibrillation and cerebral infarction were incorrectly diagnosed as having cerebral haemorrhage. It is concluded that if anticoagulation is proposed, clinical diagnosis with the GHSDS is not sufficiently accurate. However when the consequences of misdiagnosis are less important the GHSDS is useful in the clinical diagnosis of stroke if CT scanning is not available.

MICROCIRCULATORY CHANGES CORRELATING WITH CT CONTRAST ENHANCEMENT IN

CASES OF CEREBRAL INFARCTION

Nigel M Hyman. Radcliffe Infirmary Oxford

A post-mortem injection technique using barium sulphate has been applied to a group $\overrightarrow{0}$ of stroke patients who had previously, in life, undergone computed tomography. The fixed $\vec{\omega}$ brains were then cut in the same plane as that produced by the CT and the slices radio- $\bar{J}$ graphed using a conventional $x$-ray tube. The post-mortem injection and histological is examination in cases of cerebral infarctiont o revealed changes, after two to three week which included prominence of the cortic $\mathrm{g}$ palisade, arteriolar hyperplasia, venule dis్응 tension and minimal injectant extravasation The significance of these findings in re lationship to contrast enhancement on $\mathrm{C} \Phi$ following cerebral infarction is discussed.

Old infarctions failed to reveal these mI $\vec{\imath}$ crocirculatory changes but instead displaye tangled cortical vessels and corkscrew medullary vessels.

IDENTIFICATION OF THE SOURCE OF EMBOLISM IN A PATIENT WITH A MINOR STROKE USING COMPUTERISED IMAGE ANALYSIS OF CAROTID ANGIOGRAMS AND ${ }^{111}$ IN PLATELETS

ACF Colchester, DJ Hawkes, PS Monro. Atkinson Morley's Hospital, London

Improved identification of the possible source and type of embolism in individual patients with TIA or minor stroke is essential if rational decisions are to be made about endarterectomy or drug therapy. Approximate estimates of degree of carotid stenosis 3 using ultrasound or visual assessment of an-

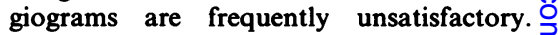
More detailed visual analysis of angiograms may allow tentative identification of mural 을 thrombus or ulceration ${ }^{1}$ but is unreliable. Gamma camera imaging of the neck following injection of ${ }^{111}$-In labelled autologous platelets may help to confirm the presence of $\widetilde{N}$ mural thrombus ${ }^{2}$ but requires further clin- $N$ ical evaluation. We are assessing methods of .



.

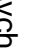

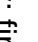
. 
computerised analysis of biplane carotid angiograms and ${ }^{111}$-In platelet studies in helping to identify sources of active embolisation, and we describe a case which illustrates the potential role of these techniques.

A 64 year-old-man presented with left monocular visual disturbance and right hemiparesis. Multiple highly refractile emboli were observed in the left retinal vessels. There was significant local uptake of labelled platelets in the region of the left carotid bifurcation. Angiography revealed stenosis of the proximal left internal carotid with different degrees of apparent narrowing in the AP and lateral views. These appearances were quantified and the data synthesised using a computerised 3-D reconstruction technique which also allowed acute comparison of angiographic and nuclear medicine images. At endarterectomy a $2 \mathrm{~cm}$ long section of intraluminal thrombus, which had only a small area of mural attachment near the bifurcation, was removed from the internal carotid.

\section{References}

${ }^{1}$ Eikelboom BC, Riles TR, et al. Stroke 1983;14:882-5.

${ }^{2}$ Goldman M, Leung JO, et al. Stroke 1983;14:752-6.

PROGNOSTIC VARIABLES FOR CEREBRAL AND CARDIOVASCULAR EVENTS AFTER TRANSIENT ISCHAEMIC ATTACKS OR MINOR STROKES UK-TIA Study Group

By late 1984, entry data together with an average of just over two years follow-up, were available on 2028 patients who had been randomised in the UK-TIA Aspirin Trial. This trial is of longterm aspirin in patients with transient ischaemic attacks (TIA) or minor ischaemic stroke that recover within one week. Recruitment is continuing and the treatment allocation has not yet been revealed.

The number of patients whose death has been attributed to heart disease (mostly ischaemic) exceeds the total number disabled by, or dead from stroke (109 versus 73 respectively). Of the prognostic variables recorded at randomisation, by far the most important for predicting subsequent stroke was the blood pressure; also relevant were residual neurological signs, age, peripheral vascular disease, increasing frequency of TIA, and possibly an abnormal cerebral angiogram. In contrast, for prediction of really serious vascular events (disabling stroke and/or any vascular death) the chief prognostic factors were age, angina and a history of myocardial infarction. Blood pressure was of little importance. Other relevant factors include peripheral vascular disease, cardiomegaly, diabetes, a previous stroke, cardiac failure, smoking, and possibly an abnormal cerebral angiogram.

Although these results are based on a larger series of TIA-patients than any other, they are still preliminary in the sense that follow-up is continuing. However, the extra patient years of follow-up are unlikely to alter substantially our conclusion that the prevention of cardiovascular events is of even greater importance than the prevention of stroke, but they may modify the present ranking of some of the prognostic variables.

MEMORY IMPAIRMENT FOLLOWING

HIPPOCAMPAL DAMAGE IN EARLY CHILDHOOD John Oxbury, Susan Oxbury. Department of Neurology, The Radcliffe Infirmary, Oxford.

Ammons horn sclerosis (AHS) is a severe depletion of pyramidal neurons in the $\mathrm{H}_{1}$ and end-folium zones of the hippocampus and of dentate gyrus granule cells. When it occurs in epileptic patients, it is usually unilateral and a consequence of an earlychildhood convulsion. It provides an excellent opportunity to compare the effects of chronic left and right hippocampal damage on memory.

We have examined verbal and non-verbal memory in 13 AHS patients and in 14 patients whose excised hippocampus was normal, or only mildly depleted of neurons, both pre-operatively and six months after temporal lobectomy, which included $2-3 \mathrm{~cm}$ of hippocampus, carried out en bloc thus allowing histological examination. All patients were left hemisphere languagedominant. Tests included: WAIS, paragraph recall immediate and delayed, paired associate learning and retention, and maze learning.

Findings pertinent to verbal memory were: 1. Pre-operatively both left and right AHS groups were significantly impaired relative to the "normal" hippocampus groups.

2. Operations produced marked deterioration when "normal" left hippocampus was removed. Significant, but less marked, deterioration followed removal of "normal" right hippocampus or of a left temporal lobe containing AHS. Removal of right temporal lobe containing AHS did not produce any significant deterioration.

3. Paragraph recall and paired associate learning tests may measure different left hemisphere functions, the former dependent on hippocampus and the latter on temporal neocortex.

Non-verbal memory impairment was de- tected on maze learning post-operativel $\$$ only after removal of "normal" hippoô campus, the defect being greater after right lobectomy.

The findings show that the left/right $\mathrm{d} \frac{\overline{5}}{\bar{\delta}}$ chotomy for verbal/non-verbal memory is relative rather than absolute and they raise the possibility that reorganisation of the anatomical substrate of memory following hippocampal damage in early childhoo\& may differ according to laterality.

ORNITHINE TRANSCARBAMYLASE DEFICIENCY HYPERAMMONAEMIA - LATE ONSET VARIET IB Sardharwalla, JE Wraith, MJ Noronha? Willink Biochemical Genetics Unit, Royg Manchester Children's Hospital, Marf chester

In the commonest form of ornithine transcarbamylase deficiency hyperammonaemien, a disorder of urea cycle transmitted as se linked dominant, the affected male dies within the first few days of birth. However in the rare form of the disorder, the symp toms of hyperammonaemia may manifest childhood. This paper describes such a case

A 5-year-old male child was admitted to hospital in a comatose state. Biochemicatio vestigations revealed hyperammonaemia (blood ammonia $490 \mu \mathrm{mol} / \mathrm{l}$ ) and ontge aciduria. The history revealed several क्षे vious episodes of clouding of consciousfess, vomiting, ataxia and slurred speech in sisciation with trivial infections. Despiter if tensive resuscitative measures, the died. A liver biopsy taken within șxy minutes of death revealed markedly red activity of the enzyme ornithine trañscarbamylase $-10 \%$ of control.

Family screening showed that a younge male sibling developed marked orotic acid uria following an infection and subsequent suffered several acute episodes of hypes ammonaemia, each requiring treatment wi sodium benzoate. He is maintained on a lo $\overline{\bar{H}}$ protein diet, containing 1.5 to $1.8 \mathrm{~g}$ 近 protein $/ \mathrm{kg} / \mathrm{day}$, and sodium benzoate 75 mg three times a day and is developing noo mally, both physically and mentally.

In conclusion, early recognition of the dis order is important because it can be treated successfully.

\section{SUB CORTICAL DEMENTIA}

D Dodwell, JS Snowden, B Northen, क्? Neary. Manchester Royal Infirmary

The concept of "sub-cortical dementia' 앙veloped from a description of the neurs psychological syndrome of progressio supranuclear palsy (PSP) and has also beßg applied to Huntington's chorea, Parkinson? disease and multi-infarct dementia. The tenfo 
has been criticised as misleadingly denoting pathological lesions exclusive to the subcortex and manifesting as a distinct neuropsychological syndrome. Psychological test - batteries have failed either to demonstrate the presence of dementia in these conditions or to differentiate between patients thought to have "cortical" as opposed to "subcortical" disease.

In this study mental test performance was compared in patients with PSP and biopsyproven Alzheimer's disease. Distinct neuropsychological profiles differentiated the two groups. Patients with PSP, unlike those with Alzheimer's disease, preserved the symbolic meaning of words and numerals and could identify, locate and manipulate objects in space. However, they were slow and failed on tasks requiring the effortful production of a sequential series of steps in problem solving. Cognitive impairment was not explicable in terms of the degree of opthalmoplegia.

The cognitive impairment of Alzheimer's disease predominantly reflects cerebral cortical disorders. Pathological change is exclusively subcortical in PSP suggesting that the neuropsychological differences of PSP and Alzheimer's disease are anatomically based.

THE SYNDROME OF KARL LUDWIG KAHLBAUM MP Barnes, M Saunders. Department of Neurology, Middlesbrough General Hospital

Catatonia was first described by Karl Ludwig Kahlbaum in 1868 . The term did not become well recognised until Emil Kraepelin and Eugen Bleuler introduced catatonia as a sub-type of schizophrenia in the early years of this century. There is still a tendency to consider catatonia primarily as a psychiatric illness despite many case reports illustrating the wide variety of medical, neurological and psychiatric causes.

We present our experience of the catatonic syndrome in the Department of Neurology - at Middlesbrough General Hospital over the twelve year period 1972-1984. During this time 25 cases have been seen. In only one patient was the syndrome associated with schizophrenia. In five patients $(20 \%)$ there was a clear-cut organic cause including two cases of encephalitis and cases of multiple sclerosis, tuberculous meningitis and a case occurring post-operatively following removal of a cerebellar haemangioblastoma. Nine patients $(36 \%)$ developed catatonia in association with affective illness. There was a marked familial tendency in this group to develop both depression and catatonia simultaneously and recurrent episodes were common. Ten patients $(40 \%)$ had no identifiable pathology either psychiatric or organic. In these cases, nine of whom were female, there was also a marked risk of recurrence. occasionally there was an association with epilepsy and in some patients there was a family history of a similar disorder. Five patients, (three in the affective group and two in the idiopathic group), presented with the acute and rapidly progressive form of catatonia and four of these people died in acute renal failure. The remainder of the patients in this series all made excellent recoveries, either spontaneously or following ECT.

We wish to emphasise that catatonia is a non-specific syndrome with a wide variety of organic and psychiatric as well as undetermined causes.

\section{OSCILLOPSIA AND RETINAL IMAGE}

STABILISATION IN PATIENTS WITH NYSTAGMUS DN Rushton. MRC Neurological Prostheses Unit, Institute of Psychiatry, London

Oscillopsia can occur when abnormal eye movements cause unintended displacement of the retinal image. However, oscillopsia can become less with time although the movements remain. This presumably occurs by central adaptation, perhaps via outflow monitoring. Such compensation is probably akin to part of the mechanism for stabilisation of the visual world that is seen in normal saccadic and fast-phase (but not slow) eye movements. However, in patients with persisting oscillopsia, such compensation must be absent or incomplete.

A simple wearable optical device for obtaining a stabilised retinal image has been described. ${ }^{1}$ By varying the power of the spectacle and contact lenses of the device, the amount of retinal image stabilisation can be varied up to about $90 \%$, using normal lens materials. When applying this device in patients with oscillopsia, it is therefore necessary to assess how much central compensation is occurring, and arrange for sufficient retinal image stabilisation to cancel the remaining oscillopsia, a rough estimate may be obtained at the bedside by comparing the amplitude of nystagmus in terms of optic disc diameters with the amplitude of oscillopsia described by the patients in terms of diameters of an object the size of the blind spot. Alternatively, different amounts of stabilisation can be tried, to minimise oscillopsia, but this requires a large trial set of contact lenses.

\section{References}

${ }^{1}$ Rushton DN, Rushton RH. J Physiol (Lond) 1984:357, 3P.
NEUROMYELITIS OPTICA; AN ANALYSIS AND FOLLOW UP OF 12 CASES

SS Pollock, CP Binning. Manchester Royal Infirmary

Neuromyelitis optica (NO) is characterised by the acute development of optic neuritis with spinal cord involvement. Despite its reported occurrence at the extremes of life and atypical findings in the cerebrospinal fluid it is commonly regarded as a variant of multiple sclerosis. In order to test this assumption we have identified the 12 patients seen in the Department of Neurology over the period 1954 to 1984 in whom a discharge diagnosis of NO was made. There was no seasonal or geographical variation but temporal clustering was apparent. Three quarters of the cases were female and none were over 34 years old. Spinal involvement normally followed visual loss and varied more widely in degree. The immediate prognosis which was good in the majority of cases did not relate to the findings in the cerebrospinal fluid but showed a clear relationship with age at onset.

Satisfactory follow up has been achieved on patients. Death from progression of the myelitis occurred in three patients. Twoण o other patients reported further episodes of reversible visual loss but we have been un $\mathbb{D}$ able to identify evidence of progression tō 음 other features of multiple sclerosis in any of $\triangle$ the patients in a follow-up period ranging $c$ from 1-28 years (mean 15.7 years).

CSF MYELIN BASIC PROTEIN IN MULTIPLE SCLEROSIS

Alan J Thompson, J Brazil, EA Martin, Feighery, M Hutchinson. Department of Neurology, St Vincent's/Adelaide Hospitals. Department of Immunology, St James's Hospital, Dublin

Cerebrospinal fluid (CSF) from 221 patients with multiple sclerosis (MS) and 85 patients with other neurological disorders (OND) was examined using a competitive radioimmunoassay for myelin basic protein immunoreactivity (MBP). MBP was found in 46 of 55 MS patients ( $84 \%$ ) examined within six weeks of relapse but in only 11 of 85 patients $(13 \%)$ with OND. There was a significant correlation between the concentration of MBP in the CSF and relapse severity in patients seen within four weeks of the onset of symptoms $(p<0.01)$. Of 44 patients in remission, MBP was detected in 12, and these patients had a significantly higher tendency to subsequent relapse $(p<0.05)$. In 72 patients with progressive disease the presence of MBP in the CSF reflected the confidence of clinical diagnosis. 
The results of this study suggest that measurement of MBP in the CSF gives an objective method of monitoring disease activity in patients with multiple sclerosis.

HYPERBARIC OXYGEN AND MULTIPLE SCLEROSIS, RESULTS OF A PLACEBOCONTROLLED, DOUBLE-BLIND TRIAL MP Barnes, D Bates, NEF Cartlidge, DM French, DA Shaw. Department of Neurology, Royal Victoria Infirmary, Newcastle upon Tyne

There has recently been considerable interest in the use of hyperbaric oxygen for multiple sclerosis. Several uncontrolled studies and one controlled trial have shown beneficial effect on the disease. Larger scale controlled trials have been needed to confirm these findings. We have studied 120 patients with chronic multiple sclerosis who were divided into two matched groups. One group received $100 \%$ oxygen at two atmospheres absolute for 90 minutes daily for a total of 20 exposures. The placebo group received normal air at normal pressure for the same length of time within the same compression chamber.

At the end of treatment no patient had improved in either group on the Kurtzke disability status scale. Improvement occurred on the Kurtzke functional systems scale only on the subjective bowel/bladder parameter, $(p=0.0338)$. Assessment at six months has failed to show any evidence of later improvement nor has there been any slowing of disease progression. Analysis of our objective tests (evoked potentials, urodynamic assessment and lymphocyte surface markers) has also failed to demonstrate any benefit from the treatment. However, the amplitude of the visual evoked response significantly decreased during the course of the therapy. This change was not sustained at six months. We believe that this may represent a reversible degree of retinal damage induced by the hyperbaric oxygen.

The short term results of this trial do not support the claims made for hyperbaric oxygen in the management of multiple sclerosis.

BOTULINUM TOXIN TREATMENT OF THE BLEPHAROSPASM-OROMANDIBULAR DYSTONIA SYNDROME

JS Elston, RW Ross Russell. Moorfields Eye Hospital and National Hospital, London

Botulinum Toxin A (BTXA) creates a temporary localised flaccid paralysis after injection into skeletal muscle. 34 patients with the blepharospasm-oromandibular dystonia syndrome have been treated with injections of BTXA into the orbicularis oculi, 28 being functionally improved by the treatment.
There was a high incidence of local side effects, especially partial ptosis which was well tolerated. There were no systemic side effects. The average period of relief was 2.5 months, increasing to 2.8 months after a second injection. Functional improvement was limited in patients with severe associated dystonia.

TRIGEMINAL SENSORY NEUROPATHY, CONNECTIVE TISSUE DISEASE AND AUTOIMMUNITY

BRF Lecky, RAC Hughes, NMF Murray. The National Hospitals and Guy's Hospital, London

Clinical and electrophysiological findings in 21 patients with chronic trigeminal sensory neuropathy are presented. The main clinical features were a slowly evolving unilateral or bilateral facial numbness sometimes associated with pain or paraesthesiae and commonly with disturbed taste. Nine patients had either systemic sclerosis or mixed connective tissue disease. Of the 12 patients, eight had either organ or non-organ specific serum autoantibodies.

Blink reflex latencies to supraorbital nerve stimulation were recorded in 17 patients. The commonest abnormality was an "afferent" defect although some responses were either normal or showed non-specific abnormalities of the $R_{2}$ components. Trigeminal sensory evoked responses were recorded in 14 cases. Six showed prolonged latencies. Other responses were either normal or degraded.

It is suggested that the lesion in this type of trigeminal neuropathy is in the trigeminal ganglion or in the proximal part of the trigeminal divisions. This conclusion is supported by limited pathological data. Possible mechanisms include vasculitis and perineurial thickening.

DECISION-MAKING IN THE MANAGEMENT OF REFRACTORY EPILEPSY: AN ANALYSIS BASED ON COMPUTER SIMULATION

Christopher D Ward. Radcliffe Infirmary, Oxford

A programme has been developed to simulate management of patients with recurrent seizures. It supplies a record of seizure dates, information about clinical toxicity and (optionally) an anticonvulsant plasma level. A Poisson process determines the occurrence of seizures with a mean frequency which can in some patients be reduced by increasing doses of anticonvulsant. The performance of 13 experienced clinicians, including 7 consultants, has been analysed. Over-reliance on a quoted plasma level therapeutic range was suggested by the high rate of requests for plasma levels, while reversible clinical to icity was rarely used as an index of max imum tolerated dose. One identifiable source of error was misinterpretation of random patterns, frequently leading to a mistake impression of non-compliance or nof random clustering. Conservative statistic criteria showed that at least $24 \%$ of followe up intervals were too short or too lons: Purely empirical considerations were fre quently subordinated to extraneous factors. The median optimum carbamazepine do $\overline{s e}$ selected for one patient was $800 \mathrm{mg}$ when the initial (arbitrary) dose was $200 \mathrm{mg}$ and 140 $\mathrm{mg}$ when the initial dose was $1200 \mathrm{mg}$. The majority of clinicians failed to perceive the in one patient the anticonvulsant was nof influencing seizure frequency. Similar erro were observed in 11 clinically inexperience subjects.

THE PATTERN OF REMISSION PERIODS IN EPILEPSY AND THEIR RELATION TO LONG-TER PROGNOSIS

SD Shorvon, JWAS Sander. Institute Neurology and Chalfont Centre for Ejlepsy.

The natural history of epilepsy has rece्बived remarkably little attention. A useful mähod of analysis is the study of the pattern of gemission periods (defined as seizure free mtervals of 2 years) in relation to the longiturinal seizure history. We report here on thespatterns of remission in two patient group 8 patients with a history of at least one-nnonfebrile epileptic seizure at some point in ${ }^{3}$ inde, identified from a general practice population of 6,000 persons; and 181 neurological of patients with chronic active epilepsy, had been followed for at least 2 years from the onset of the seizures. In both groups the niedian follow up was 17 years. All patiefts were personally interviewed and the occ घrrence and timing of remission periods an ysed in relation to the temporal course of epilepsy, and to other clinical features. Most of the patients identified from the general practice population had entered long-terim remission, usually early in the course of their seizures, and relapse following remisstion was unusual. Conversely, in the chronic $\overline{\text { Ra }}$ tients, a history of remission at any poinfin time was surprisingly rare. In both grougs, the epilepsy appeared to run 'true to forin * from its onset, and long term prognogis could often have been predicted early in the course of the epilepsy. This has obvious intplications for treatment, and also raises the possibility that early treatment may not ony suppress the seizures in the short term but actually prevent their long-term recurreres. 